Agrotrópica 32(1): 51-58. 2020.

Centro de Pesquisas do Cacau, Ilhéus, Bahia, Brasil

\title{
ACAROLOGIA AGRÍCOLA NO ESTADO DO AMAPÁ: PANORAMA HISTÓRICO E DESAFIOS FUTUROS
}

\author{
Rodrigo Souza Santos ${ }^{1}$, Jeferson Luiz de Carvalho Mineiro ${ }^{2}$, Maria do Socorro Miranda de \\ Sousa $^{3}$, Ezequiel da Glória de Deus ${ }^{4}$, Ricardo Adaime ${ }^{5}$
}

\begin{abstract}
${ }^{1}$ Embrapa Acre, Rodovia BR 364, km 14, CP 321, 69900-970, Rio Branco, Acre, Brasil. rodrigo.s.santos@embrapa.br; ${ }^{2}$ Instituto Biológico, Laboratório de Acarologia, Rodovia Heitor Penteado, km 3, 13092-541, Campinas, São Paulo, Brasil. jefmin@ hotmail.com; ${ }^{3}$ Universidade Federal do Amapá (Unifap), Programa de Pós-Graduação em Biodiversidade Tropical, Rodovia Juscelino Kubitschek, km 02, 68903-419, Macapá, Amapá, Brasil. socorro-ap@ homail.com; ${ }^{4}$ Instituto Federal do Amapá (Ifap), Rua Nilo Peçanha, nº 1.263, 68920-000, Laranjal do Jari, Amapá, Brasil. egd_bio@ @otmail.com; ${ }^{5}$ Embrapa Amapá, Rodovia Juscelino Kubitscheck, km 5, no 2600, CP 10, 68903-419, Macapá, Amapá, Brasil.

ricardo.adaime@embrapa.br
\end{abstract}

No estado do Amapá, integrante da Amazônia Legal, até o momento os estudos na área de Acarologia Agrícola foram pontuais embora tenham indicado rica diversidade de espécies. Neste trabalho apresentamos o estado da arte da Acarologia Agrícola no Amapá, com o intuito de atrair e estimular a fixação de acarologistas no Estado, assegurando a produção de conhecimento científico sobre este importante grupo biológico.

Palavras-chave: Acari, Arachnida, diversidade, praga, Amazônia.

\section{Agricultural acarology in the state of Amapá, Brazil: historical overview and}

future challenges. In the state of Amapá, Brazil, part of the Legal Amazon, so far studies in the area of Agricultural Acarology have been punctual, although they have indicated rich species diversity. In this paper we present the state of the art of Agricultural Acarology in Amapá state, in order to attract and stimulate the establishment of acarologists in the state, ensuring the production of scientific knowledge about this important biological group.

Key words: Acari, Arachnida, diversity, pest, Amazon. 


\section{Introdução}

O estado do Amapá faz parte da Amazônia Legal, possui área física de $142.817 \mathrm{Km}^{2}$ distribuídos em 16 municípios e fronteira com a Guiana Francesa ao norte e o Suriname a noroeste. O Estado possui a maior parte do seu território situado no Hemisfério Norte, sendo que a Linha do Equador passa pela capital, Macapá, no sudeste do Estado (Jordão e Silva, 2006; Só Geografia, 2019).

Os ecossistemas do Estado são diversos, existindo florestas de terra firme, campos e florestas de várzea, cerrados, florestas de transição e mangues (Costa Neto e Silva, 2004). Predominam pequenas propriedades agrícolas providas de mão-de-obra familiar, raramente com área superior a quatro hectares. Os sistemas agrícolas são dependentes da diversidade dos ecossistemas, mas a agricultura é praticada majoritariamente em ambientes de terra firme ou na parte alta das superfícies, frequentemente alagadas (Jordão e Silva, 2006).

Quanto mais distante da capital do Estado, maior a influência da agricultura como fonte de renda. A incidência de pragas e doenças, no entanto, é muito alta. As condições climáticas, aliadas às precárias condições socioeconômicas, propiciam a ocorrência de insetos, patógenos, nematoides e ácaros, resultando na redução da produção agrícola e, consequentemente, na qualidade de vida de seus agricultores (Jordão e Silva, 2006). Segundo Morais et al. (2016), os Estados da Amazônia Legal tem sido uma importante porta de entrada de pragas exóticas no Brasil, principalmente de países vizinhos. Assim, a detecção precoce de pragas é uma ferramenta chave para a adoção de medidas de contenção, as quais evitarão ou retardarão sua disseminação para novas áreas.

Os ácaros são organismos diminutos, que habitam os mais diferentes ambientes e correspondem ao segundo maior grupo de artrópodes, depois dos insetos. São comuns no meio aquático, sobre plantas e animais, assim como nos depósitos de alimentos, abrigos de animais e residências humanas. No Brasil, entre 20 a 30 espécies de ácaros causam sérios danos a plantas cultivadas, justificando o interesse e a necessidade de se conhecer essas espécies (Moraes e Flechtmann, 2008).
O conhecimento sobre as espécies de ácaros que ocorrem em cada região brasileira, seus hábitos, biologia e inter-relações ecológicas é de fundamental importância para detectar espécies potencialmente danosas aos cultivos regionais e, assim, evitar ou minimizar perdas agrícolas. Ademais, estudos de levantamento faunístico podem resultar no registro de espécies promissoras como inimigas naturais de pragas, com potencial para serem utilizadas em programas de controle biológico aplicado (AguiarMenezes et al., 2007; Moraes e Flechtmann, 2008; Nuvoloni et al., 2014; Barbosa et al., 2017), assim como indicar plantas hospedeiras que funcionem como reservatório dessas espécies e que podem ter importância em programas de controle biológico conservativo (Moraes et al., 2001; Lofego, Moraes e Castro, 2004; Lofego et al., 2017).

A Acarologia Agrícola, ciência que se ocupa do estudo dos ácaros que mantém interação com as plantas cultivadas, ainda é pouco desenvolvida no estado do Amapá. Não há acarologistas de formação atuando profissionalmente no Estado. Tampouco, existem cursos de pós-graduação que ofertem a disciplina de Acarologia. Em consequência, são escassos os trabalhos envolvendo ácaros plantícolas e predadores no estado do Amapá, tendo sido realizados apenas trabalhos pontuais, de curta duração e, em grande parte, publicados na forma de resumos (Gazel Filho, Silva e Lima, 2002; Gazel Filho e Silva, 2003; Silva e Jordão, 2005; Jordão e Silva, 2006; Mineiro, Silva e Silva, 2009; Jordão, Noronha e Funi, 2011; Deus et al., 2012; Sousa et al., 2013; Mineiro et al., 2014; Demite et al., 2015).

Nesse sentido, este trabalho reúne informações sobre as pesquisas na área da Acarologia Agrícola realizadas no estado do Amapá, com informações sobre as espécies plantícolas e predadoras registradas até o momento, bem como visa discutir os principais desafios de pesquisa nessa área no Estado.

As bases de dados consultadas foram: Plataforma Scielo, Portal de Periódicos Capes e Ainfo - Sistema de Gestão do Acervo Documental e Digital da Embrapa. Foram consultados capítulos de livros, artigos/notas científicas publicadas em periódicos indexados, boletins de pesquisa, circulares e comunicados técnicos, documentos da série Embrapa, bem como resumos publicados em Anais de eventos científicos. 


\section{Estudos acarológicos preliminares no estado do Amapá}

Nesse trabalho de revisão, verificou-se um total de 51 espécies de ácaros (fitófagos e predadores), distribuídos entre 18 famílias (Eriophyidae, Tenuipalpidae, Tetranychidae, Tarsonemidae, Phytoseiidae, Ascidae, Cunaxidae, Stigmaeidae, Tydeidae, Wintershmidtiidae, Blattisociidae, Melicharidae, Oripodidae, Acaridae, Diptilomiopidae, Echimyopodidae, Eupodidae e Iolinidae) em 13 espécies de plantas hospedeiras no estado do Amapá. Os ácaros plantícolas mais importantes registrados até o momento no estado ao Amapá estão relacionados na Tabela 1. Considerando a amplitude de ocorrência e danos causados, destacam-se: Brevipalpus yothersi Baker (Acari: Tetranychidae) e Plyllocoptrutra oleivora (Ashmead) (Acari:Eriophyidae) em Citrus sinensis (L.) Osbeck (Rutaceae). Embora Tetranychus abacae Baker \& Pritchard (Acari: Tetranychidae) seja muito frequente em Musa sp. (Musaceae), não foram observados danos significativos à cultura até o momento.

Os trabalhos pioneiros de Mineiro, Silva e Silva (2009), Deus et al. (2012), Sousa et al. (2013) indicaram significativa riqueza de espécies de ácaros predadores, que por sua vez, são potenciais agentes no controle biológico natural dos ácaros-praga.
Os primeiros relatos de ácaros de importância agrícola no estado do Amapá foram realizados por Gazel Filho, Silva e Lima (2002). Os autores relataram o ácaro-da-necrose, Aceria (Eriophyes) guerreronis Keifer (Acari: Eriophyidae), em coqueiros (Cocos nucifera L., Arecaceae) no município de Macapá. A espécie é considerada praga-chave do coqueiro no Brasil, especialmente na região Nordeste (Ferreira et al., 1998). No Amapá, onde a produção é voltada exclusivamente para o consumo de água, essa praga pode comprometer seriamente o cultivo do coco, visto que os frutos atacados por A. guerreronis podem ter uma redução de $50 \%$ no tamanho, quando comparados a frutos sadios (Moura e Leite, 2000).

Posteriormente, Gazel Filho e Silva (2003) relataram o ácaro-da-mancha-anelar-do-coqueiro, Amrineus cocofolius Flechtmann (Acari: Eriophyidae), em plantio comercial de coco no estado do Amapá. Tal espécie forma uma banda necrótica transversal no fruto, superficialmente, sem formar rachaduras (Navia et al., 2005).

Silva e Jordão (2005) relataram a ocorrência do ácaro-da-falsa-ferrugem, P. oleivora, e do ácaro-daleprose, Brevipalpus phoenicis (Geijskes) (Acari: Tenuipalpidae) em $C$. sinensis, informando que possuem ocorrência generalizada e causam danos significativos. Mineiro et al. (2009) reportaram o ácaro-mexicano, Tetranychus mexicanus (McGregor) (Acari:

Tabela 1. Principais espécies de ácaros plantícolas registradas no estado do Amapá e suas respectivas plantas hospedeiras

\begin{tabular}{|c|c|c|c|}
\hline Espécies de ácaros & Famílias & Espécies vegetais & Referências \\
\hline $\begin{array}{l}\text { Brevipalpus yothersi Baker* } \\
\text { Tenuipalpus heveae Baker } \\
\text { Tenuipalpus uvae De Leon }\end{array}$ & Tenuipalpidae & $\begin{array}{l}\text { Citrus sinensis (Rutaceae) } \\
\text { Hevea brasiliensis (Euphorbiaceae) } \\
\text { Spondias mombin (Anacardiaceae) }\end{array}$ & $\begin{array}{l}\text { Silva e Jordão (2005) } \\
\text { Jordão e Silva (2006)** } \\
\text { Mineiro et al. (2009) } \\
\text { Deus et al. (2012) } \\
\text { Mineiro et al. (2014) }\end{array}$ \\
\hline $\begin{array}{l}\text { Mononychellus tanajoa (Bondar) } \\
\text { Tetranychus abacae Baker \& Pritchard } \\
\text { Tetranychus mexicanus (McGregor) }\end{array}$ & Tetranychidae & $\begin{array}{l}\text { Manihot esculenta (Euphorbiaceae) } \\
\text { Musa sp. (Musaceae) } \\
\text { Citrus sinensis (Rutaceae) }\end{array}$ & $\begin{array}{l}\text { Mineiro et al. (2009) } \\
\text { Jordão e Silva (2006) } \\
\text { Mineiro et al. (2009) }\end{array}$ \\
\hline Polyphagotarsonemus latus (Banks) & Tarsonemidae & Carica papaya (Caricacae) & Jordão e Silva (2006) \\
\hline Davisella spondias Reis \& Navia & Diptilomiopidae & Spondias mombin (Anacardiaceae) & Sousa et al. (2013) \\
\hline Phyllocoptruta oleivora (Ashmead) & Eriophyidae & Citrus sinensis (Rutaceae) & $\begin{array}{l}\text { Silva e Jordão (2005) } \\
\text { Mineiro et al. (2009) }\end{array}$ \\
\hline
\end{tabular}


Tetranychidae), associado ao cultivo de $C$. sinensis no estado do Amapá.

Phyllocoptruta oleivora é reportado como a principal praga dos citros no mundo (Yamamoto e Bassanezi, 2003). No Brasil, é a segunda espécie de ácaro-praga mais relevante, sendo $B$. phoenicis o principal ácaro-praga dos citros no Brasil, por ser o transmissor do vírus-da-leprose e também do vírus-daclorose-zonada (Moraes e Flechtmann, 2008).

Jordão e Silva (2006), após levantamento de pragas em alguns municípios do Amapá, relataram a ocorrência das seguintes espécies de ácaros: o ácaro-vermelho, $T$. abacae em bananeira, além do ácaro-branco, Polyphagotarsonemus latus (Banks) (Acari: Tarsonemidae) e do ácaro-de-teia, Tetranychus sp. (Acari: Tetranychidae), em mamoeiro (Carica papaya L., Caricaceae).

Mineiro, Silva e Silva (2009) realizaram um levantamento de espécies de ácaros que ocorrem em fruteiras e outras plantas de importância econômica nos municípios de Macapá e Calçoene. Os autores registraram espécimes das famílias Ascidae, Phytoseiidae, Cunaxidae, Eriophyidae, Stigmaeidae, Tarsonemidae, Tenuipalpidae, Tetranychidae e Tydeidae, das quais a família Tetranychidae apresentou a maior riqueza (cinco espécies). Dentre as espécies coletadas por Mineiro, Silva e Silva (2009), destacouse Mononychellus tanajoa (Bondar) (Acari: Tetranychidae), apontada por Moraes e Flechtmann (2008) como importante praga da cultura da mandioca (Manihot esculenta Crantz, Euphorbiaceae).

Jordão, Noronha e Funi (2011) fizeram levantamento de ácaros em mandioca nos municípios de Macapá, Calçoene e Oiapoque. Foram obtidos espécimes pertencentes às famílias Acaridae, Cheyletidae, Phytoseiidae, Tarsonemidae e Tetranychidae. Embora em todas as amostras tenham sido coletados espécimes de Mononychellus sp. (Acari: Tetranychidae), os autores não observaram danos significativos às plantas.

\section{Considerações sobre Brevipalpus phoenicis (Geijskes)}

Ochoa et al. (2011) observaram a existência de diferentes morfoespécies do ácaro-plano, $B$. phoenicis, até então consideradas como uma única espécie. A partir disso foram iniciados alguns estudos morfológicos evidenciando essas diferenças (Ferreira et al., 2013; Mineiro et al., 2013a, 2013b, 2014, 2015a, 2015b; Ochoa et al., 2015; Sinico et al., 2015), ficando caracterizado o chamado "complexo B. phoenicis". Posteriormente, Beard et al. (2013) separaram essas morfoespécies em distintas espécies.

Assim, durante mais de 70 anos de estudos do ácaroplano no Brasil, houve um aumento expressivo de trabalhos científicos envolvendo essa espécie desde a década de 90, principalmente devido à sua importância econômica e às novas descobertas sobre essas espécies de ácaros atuarem como transmissores de vírus para plantas. Quatro espécies novas foram descritas, incluindo muitas espécies crípticas dentro do grupo $B$. phoenicis. Duas sinonímias de $B$. phoenicis lato sensu, B. yothersi e Brevipalpus papayensis Baker (Acari: Tenuipalpidae), foram ressuscitadas e redescritas (Beard et al., 2015). Para isso, foram utilizados outros caracteres morfológicos que foram observados e adicionados para a separação das espécies pelo uso de modernas técnicas de microscopia (Bolton et al., 2014).

Dessa forma, a maioria das espécies relatadas e consideradas como B. phoenicis no Brasil até então, estão equivocadas. As espécies B. phoenicis, B. papayensis e $B$. yothersi ocorrem em citros, porém, $B$. yothersi é a mais amplamente disseminada (Beard et al., 2015). Novos estudos com relação à transmissão de vírus, biologia, ecologia, sensibilidade a produtos químicos, biologia molecular, entre outros, estão sendo realizados por pesquisadores, tanto no Brasil quanto em outros países, para tentar elucidar diferentes aspectos dessa espécie de ácaro.

Diante do exposto acima, no que tange aos trabalhos realizados no Amapá, deve-se fazer as seguintes considerações:

1) Em trabalho sobre ácaros conduzidos em espécies frutíferas, Mineiro, Silva e Silva (2009) registraram B. phoenicis em citros nos municípios de Macapá e Calçoene. Entretanto, deve-se considerálo como B. yothersi.

2) Sousa et al. (2013) amostraram B. phoenicis em taperebazeiro. Porém, ao examinarem o exemplar mais detalhadamente, foi possível verificar que se tratava de outra espécie. No entanto, como foi encontrado apenas um indivíduo, não é possível confirmar a sua identificação com segurança ao nível específico ou mesmo a possibilidade de ser um táxon ainda não descrito. 


\section{Ácaros em seringueira [Hevea brasiliensis (Willd. ex Adr. de Juss.) Müell. Arg., Euphorbiaceae]}

Deus et al. (2012) realizaram o primeiro estudo sistematizado sobre os ácaros associadas à seringueira, Hevea brasiliensis (Willd. ex Adr. de Juss.) Müell. Arg., Euphorbiaceae, no estado do Amapá. O trabalho foi realizado em um seringal implantado entre os anos 1980 e 1982, no município de Santana. As coletas foram realizadas no clone Fx 2261, plantado em 1982 em área correspondente a dois hectares.

Os autores amostraram 1.722 ácaros distribuídos nas famílias Acaridae, Cunaxidae, Eriophyidae, Iolinidae, Phytoseiidae, Stigmaeidae, Tarsonemidae, Tenuipalpidae, Tydeidae e Winterschmidtiidae, além de espécies não identificadas das subordens Oribatida e Astigmata. A família Tenuipalpidae, embora representada apenas pelo ácaro-plano-vermelho-daseringueira, Tenuipalpus heveae Baker, apresentou o maior número de indivíduos capturados $(81,1 \%)$, com média de 3,1 ácaros/folíolo. Este foi o primeiro registro de T. heveae para o estado do Amapá (Deus et al., 2012). Essa espécie habita principalmente a face abaxial das folhas, causando um bronzeamento das folhas pela perda de área fotossintética, seguido de queda prematura das mesmas (Moraes e Flechtmann, 2008). Essa espécie passou a ser reportada como praga potencial da seringueira no Brasil a partir do ano 2000 (Vieira et al., 2009).

Os autores verificaram que o principal ácaro-praga no seringal estudado foi $T$. heveae, com elevado nível populacional nas duas amostragens (Deus et al., 2012). Foi verificado ainda o ataque do fungo Hirsutella sp. (Ophiocordycipitaceae) infectando espécimes de $T$. heveae, atuando como um potencial agente de controle biológico natural desta espécie no ambiente. A espécie Hirsutella thompsonii (Fisher) (Deuteromycotina: Hyphomycetes) já havia sido registrada por Tanzini et al. (2000), atacando Calacarus heveae Feres (Acari: Eriophyidae) em seringueira, em Itiquira, MT.

Calacarus heveae, importante espécie-praga da seringueira no Brasil, não foi amostrada no levantamento realizado por Deus et al. (2012). Entretanto, para confirmar a ausência da espécie no Amapá, será necessário a realização de novos levantamentos em outras áreas de seringais do estado.

\section{Ácaros em taperebazeiro (Spondias mombin L., Anacardiaceae)}

Sousa et al. (2013) realizaram o primeiro levantamento de ácaros associados ao taperebazeiro, através de amostragens quinzenais, em duas áreas experimentais estabelecidas em cultivos no município de Porto Grande.

Foram obtidos exemplares de 17 famílias nas duas áreas amostradas (Melicharidae, Phytoseiidae, Ascidae, Blattisociidae, Galumnidae, Oripodidae, Phthiracaridae, Eupodidae, Acaridae, Histiosmotidae, Cunaxidae, Diptilomiopidae, Echimyopodidae, Iolinidae, Stigmaeidae, Tarsonemidae e Tenuipalpidae). A espécie mais abundante foi Davisella spondias Reis \& Navia (Diptilomiopidae), representando mais de $90 \%$ do total de ácaros capturados nas duas áreas. Essa espécie foi descrita por Reis et al. (2010), associada a S. mombin em Recife, PE. Não foram detectadas injúrias causadas por estes ácaros à planta hospedeira (Souza et al., 2013).

Exemplares de Tenuipalpus uvae De Leon (Acari: Tenuipalpidae) foram obtidos nas duas áreas. Verificou-se que se tratava do primeiro registro dessa espécie no Brasil, juntamente com espécimes obtidos também em Pernambuco (Mineiro et al., 2014). Essa espécie causa finas rachaduras na epiderme de frutos de taperebá e amarelecimento uniforme nas folhas terminais, o que afeta a qualidade e a comercialização dos frutos (Salas e Ochoa, 1986).

Considerando a diversidade de espécies associadas ao taperebazeiro neste levantamento preliminar realizado no estado do Amapá por Souza et al. (2013), com destaque para a ocorrência de $D$. spondias e a presença da espécie nociva $T$. uvae, sugere-se a realização de levantamentos mais sistematizados, tanto em áreas de ocorrência espontânea dessa espécie vegetal quanto em áreas cultivadas.

\section{Ácaros predadores}

As principais famílias de ácaros plantícolas predadores, são Anystidae, Bdellidae, Cheyletidae, Cunaxidae, Phytoseiidae e Stigmaeidae (Yaninek \& Moraes, 1991; Gerson, Smiley \& Ochoa, 2003). Dentre os estudos acarológicos realizados no estado do Amapá, foi verificada a presença de diversas espécies de ácaros predadores, em várias espécies vegetais. 
No levantamento de ácaros realizado em cultivo de mandioca por Jordão, Noronha e Funi (2011), os fitoseídeos representaram $94 \%$ dos espécimes predadores. As espécies amostradas foram Amblyseius aerialis (Muma), Amblydromalus sp. e Typhlodromalus aripo De Leon (Acari: Phytoseiidae). Os ácaros pertencentes à família Phytoseiidae são conhecidos principalmente por seu hábito predador, embora muitos se alimentem também de pólen, fungos, substâncias açucaradas produzidas por insetos, exsudatos de plantas etc. Ocorrem principalmente sobre plantas, ainda que também encontrados no solo (Moraes e Flechtmann, 2008). Atualmente são conhecidas mais de 2.250 espécies de fitoseídeos em todo o mundo, das quais cerca de 140 já foram reportadas no Brasil (Moraes et al. 2004). Os fitoseídeos são amplamente reconhecidos como eficientes agentes utilizados em programas de controle biológico de pragas no Brasil e no mundo (Moraes, 1991; Moraes e Flechtmann, 2008; Praslièka et al., 2009).

O estudo conduzido por Demite et al. (2015) nos municípios de Amapá, Calçoene, Macapá, Oiapoque e Santana, registrou 23 espécies de fitoseídeos, sendo as espécies A. aerialis, Amblyseius largoensis (Muma) e Amblyseius tamatavensis Blommers (Acari: Phytoseiidae) as mais representativas nas amostras. Os autores verificaram que as plantas que abrigaram a maior riqueza de fitoseídeos foram Musa sp., $C$. nucifera e Euterpe oleracea Mart. (Arecaceae).

Deus et al. (2012) também amostraram famílias de ácaros que apresentam espécies predadoras, tais como Phytoseiidae, Stigmaeidae e Cunaxidae. Entre os Phytoseiidae coletados, os autores amostraram representantes dos gêneros Amblyseius, Euseius, Galendromus e Typhlodromips.

Souza et al. (2013) verificaram que a família Phytoseiidae apresentou a maior riqueza de espécies, com relação aos demais ácaros predadores amostrados, em levantamento conduzido em cultivos de taperebazeiros no município de Porto Grande. Os autores também coletaram espécimes de ácaros predadores pertencentes às famílias Cunaxidae e Stigmaeidae. Espécimes de fitoseídeos dos gêneros Amblyseius, Amblydromalus, Iphiseiodes, Phytoscutus e Proprioseiopsis foram as mais abundantes nesse levantamento. Ademais, foram amostradas duas novas espécies ainda não descritas formalmente, pertencentes aos gêneros Iphiseius e Phytoscutus (Souza et al. 2013).

Demite et al. (2015) destacaram que, devido à grande diversidade florística presente no estado do Amapá, as espécies de fitoseídeos até o momento conhecidas representam uma fração muito pequena da diversidade dessa família no Estado, reforçando a necessidade de estudos mais abrangentes e duradouros que contemplem vários municípios e tipos de vegetação (silvestre e agrícola) ainda não amostrados.

\section{Considerações Finais}

O conhecimento sobre os ácaros e as plantas a que estão associados ainda é muito limitado no estado do Amapá, sendo representado por uma escassa literatura publicada até o momento: três artigos científicos, duas notas científicas, um comunicado técnico, três resumos em eventos e algumas informações presentes nos livros publicados por Jordão e Silva (2006) e Silva, Adaime e Zucchi (2016). Esse conhecimento é resultante de estudos preliminares, porém pioneiros, realizados por pesquisadores que atuam ou atuaram no Estado, em geral com formação na área de Entomologia Agrícola. Ressalta-se que, para a geração do conhecimento até então disponível, foi crucial o apoio de experientes acarologistas radicados em outras unidades da federação.

Em condições de campo verifica-se danos causados por ácaros em algumas culturas agrícolas. No entanto, estudos específicos sobre ácaros nos cultivos mais expressivos para a população local, tais como mandioca, feijão-caupi, olerícolas e frutíferas em geral ainda não foram realizados. Esses estudos devem, inclusive, contemplar os Sistemas Agroflorestais (SAFs), modalidade de produção bastante adotada pelos agricultores no Estado. Considerando a recente expansão das áreas agrícolas no Amapá é de se esperar que os prejuízos econômicos causados por ácaros sejam intensificados. Por outro lado, identifica-se claramente fértil terreno para jovens acarologistas que desejem iniciar sua trajetória profissional no Estado.

Os poucos estudos realizados indicam significativa riqueza de espécies, especialmente de Phytoseiidae, importante família de ácaros predadores. Portanto, levantamentos de ácaros nos diversos ecossistemas 
amapaenses são prioritários. Além de estudos focados em catalogar a diversidade da acarofauna no Estado, outros, referentes à ecologia, etologia, dinâmica populacional e controle de espécies de importância econômica são também necessários.

Adicionalmente, não há corpo técnico preparado para identificar ácaros de expressão quarentenária para o Brasil, já introduzidos ou na iminência de introdução. Há que se considerar que, nos anos recentes, duas espécies de ácaros foram introduzidas no país, pela Amazônia, mais especificamente via estado de Roraima: 1) o ácaro-hindustânico-dos-citros, Schizotetranychus hindustanicus (Hirst) (Acari: Tetranychidae), em 2008 (Navia e Marsaro Júnior, 2010) e 2) o ácaro-vermelhodas-palmeiras, Raoiella indica Hirst (Acari: Tenuipalpidae), em 2009 (Navia et al., 2011). Há ainda um sério risco de introdução, também por esta região, de Steneotarsonemus spinki (Acari: Tarsonemidae), importante praga de arrozais na Ásia e América Central, e que já se encontra reportado na Colômbia (Navia, Mendonça \& Ochoa, 2010; Toro e Mesa, 2015).

Urge, pois, atrair e fixar acarologistas para atuar no conhecimento básico e aplicado sobre as espécies de ácaros que causam prejuízos econômicos aos cultivos locais e sobre os ácaros predadores que atuam no controle biológico natural das espécies nocivas.

\section{Literatura Citada}

AGUIAR-MENEZES, E. L. et al. 2007. Ácaros: taxonomia, bioecologia e sua importância agrícola. Seropédica, RJ, Embrapa Agrobiologia. Documentos, 240. 28p.

BARBOSA, M. F. de C. et al. 2017. Controle biológico com ácaros predadores e seu papel no manejo integrado de pragas. Engenheiro Coelho: PROMIP. 69p.

BEARD, J. J. et al. 2013. Flat mites of the world. $2^{\text {nd }}$ edition. Disponível em: < http://idtools.org/id/mites/ key.php?key=Brevipalpus_of_the_world_2013> Acesso em: 26 mar. 2020.

BEARD, J. J. et al. 2015. Brevipalpus phoenicis (Geijskes) species complex (Acari: Tenuipalpidae) - a closer look. Zootaxa 3944(1):1-67.

BOLTON, S. J. et al. 2014. A new genus and species for Nematalycidae (Acari: Endeostigmata). Journal of Natural History 48:1359-1373.

COSTA NETO, S. V.; SILVA, M. S. 2004. Vegetação. In: Santos, V. F.; Figueira, Z. R. (Orgs.). Diagnóstico sócio-ambiental participativo do setor costeiro estuarino do Estado do Amapá. Macapá, Amapá, MMA/GEA/IEPA. CD-ROM. pp.84-114.
DEUS, E. G. et al. 2012. Mites (Arachnida: Acari) collected on rubber trees Hevea brasiliensis (Willd. ex A. Juss.) Müell. Arg. in Santana, Amapá state, Brazil. Brazilian Journal of Biology (Brasil) 72(4):915-918.

DEMITE, P. R. et al. 2015. Ácaros fitoseídeos (Acari: Phytoseiidae) plantícolas do estado do Amapá, Brasil. In: Simpósio Brasileiro de Acarologia, 5. São José do Rio Preto. Resumos. São José do Rio Preto, SP, SIBAC.

FERREIRA, J. M. S. et al. 1998. Pragas do coqueiro. In: Ferreira, J. M. S.; Warwick, D. R. N.; Siqueira, L. A. (eds.). A cultura do coqueiro no Brasil. $2^{\text {a }}$ ed. Brasília, Embrapa - SPI; Aracaju, SE, Embrapa CPATC. pp. 189-267.

FERREIRA, P. R. et al. 2013. Distribuição de morfoespécies de Brevipalpus phoenicis do grupo C (Acari: Tenuipalpidae) em cafeeiros em diferentes localidades no Estado de São Paulo. In: Reunião Anual do Instituto Biológico, 26. São Paulo. Resumos. São Paulo, SP, RAIB.

GAZEL FILHO, A. B.; SILVA, R. A. 2003. Artrópodes prejudiciais ao cultivo do coqueiro (Cocos nucifera $\mathrm{L}$. Arecaceae) no Estado do Amapá, Brasil. In:Reunião Anual do Instituto Biológico, 16. São Paulo. Resumos... São Paulo, SP, Arquivos do Instituto Biológico. pp. 48-49.

GAZEL FILHO, A. B.; SILVA, R. A.; LIMA, J. A. de S. 2002. Pragas do coqueiro (Cocos nucifera L.) no Amapá. Macapá, Embrapa Amapá. Comunicado Técnico, 79. 9p.

GERSON, U.; SMILEY, R. L.; OCHOA, R. 2003. Mites (Acari) for pest control. Oxford: Blackwell Science. 539p.

JORDÃO, A. L.; NORONHA, A. C. S.; FUNI, C. 2011. Ácaros em folhas de mandioca (Manihot esculenta Crantz) no estado do Amapá. In: Congresso Brasileiro de Mandioca, 14; $1^{\text {a }}$ Feira Brasileira da Mandioca, Maceió. Resumos. Maceió, AL, SBM. 5p.

JORDÃO, A. L.; SILVA, R. A. 2006. Guia de pragas agrícolas para o manejo integrado no Estado do Amapá. Ribeirão Preto, SP, Holos Editora. 182p.

LOFEGO, A. C.; MORAES, G. J. de; CASTRO, L. A. S. 2004. Phytoseiid mites (Acari: Phytoseiidae) on Myrtaceae in the State of São Paulo, Brazil. Zootaxa 516:1-18.

LOFEGO, A. C. et al. 2017. Mite fauna associated with Cecropia pachystachya Trec. (Urticaceae) - the importance of the plant as reservoir and dissemination means for predatory mites. Systematic \& Applied Acarology 22(10):1780-1794.

MINEIRO, J. L. C. et al. 2014. First record of Tenuipalpus uvae De Leon, 1962 (Acari: Tenuipalpidae) in Brazil. Check List (Brasil) 10(1):151-152.

MINEIRO, J. L. C. et al. 2013a. Caracterização morfológica de diferentes morfoespécies de Brevipalpus phoenicis (Acari: Tenuipalpidae). In: Simpósio Brasileiro de Acarologia, 4. Bento Gonçalves. Resumos. Bento Gonçalves, RS, SIBAC.

MINEIRO, J. L. C. et al. 2013b. Distribuição de diferentes morfoespécies de Brevipalpus phoenicis (Acari: Tenuipalpidae) em diferentes localidades e plantas no Estado de São Paulo. In: Simpósio Brasileiro de Acarologia, 4. Bento Gonçalves. Resumos. Bento Gonçalves, RS, SIBAC. 
MINEIRO, J. L. C. et al. 2015a. Brevipalpus phoenicis no Brasil: de 2011 a 2015, o que aconteceu? In: Simpósio Brasileiro de Acarologia, 5. São José do Rio Preto. Resumos... São José do Rio Preto, SP. SIBAC.

MINEIRO, J. L. C. et al. 2015b. Brevipalpus yothersi em Citrus spp. no Brasil: Resultados preliminares. In: Simpósio Brasileiro de Acarologia, 5. São José do Rio Preto. Resumos... São José do Rio Preto: SIBAC.

MINEIRO, J. L. C. et al. 2014. Brevipalpus phoenicis (group species B) on Citrus spp. and Coffea arabica, State of São Paulo, Brazil. In: International Congress of Acarology, 14. Kyoto, Japan. Proceedings. Kyoto, Acarology Society of Japan.

MINEIRO, J. L. C.; SILVA, W. R.; SILVA, R. A. 2009. Ácaros em fruteiras e outras plantas no Estado de Amapá. Biota Neotropica (Brasil) 9(2):103-106.

MORAES, G. J. de. 1991. Controle biológico de ácaros fitófagos. Informe Agropecuário (Brasil) 15(167):56-62.

MORAES, G. J. de et al. 2001. Papel da vegetação natural como reservatório de ácaros predadores. In: Simpósio de Controle Biológico, 7. Poços de Caldas. Resumos... Poços de Caldas: Microservice - Tecnologia Digital S.A. pp. 492-497.

MORAES, G. J. de; FLECHTMANN, C. H. W. 2008. Manual de Acarologia: acarologia básica e ácaros de plantas cultivadas no Brasil. Ribeirão Preto, Holos Editora. 308p.

MORAES, G. J. de et al. 2004. A revised catalog of the mite family Phytoseiidae. Zootaxa 434:494p.

MORAIS, E. G. F. de et al. 2016. Pragas de expressão quarentenária na Amazônia. pp. 521-559. In: Silva, N. M. da; Adaime, R.; Zucchi, R. A. (eds.). Pragas agrícolas e florestais na Amazônia. Brasília, Embrapa. 608p.

MOURA, J. I. L.; LEITE, J. B. 2000. Ocorrência de pragas no coqueiro (Cocos nucifera L.) cultivado em sistema agroflorestal no sul da Bahia. In: Congresso Brasileiro de Sistemas Agroflorestais, 3. Manaus. Resumos... Manaus, AM, Embrapa Amazônia Ocidental. pp. 238-241.

NAVIA, D.; MARSARO JÚNIOR, A. L. 2010. First report of the citrus hindu mite, Schizotetranychus hindustanicus (Hirst) (Prostigmata: Tetranychidae), in Brazil. Neotropical Entomology (Brasil) 39(1):140-143.

NAVIA, D. et al. 2005. Acarofauna associada a frutos de coqueiro (Cocos nucifera L.) de algumas Localidades das Américas. Neotropical Entomology (Brasil) 34(2):349-354.

NAVIA, D.; MENDONÇA, R. S.; OCHOA, R. 2010. The rice mite Steneotarsonemus spinki, an invasive species in the Americas. In: Sabelis, M. W; Bruin, J. (eds.). Trends in acarology: proceedings. International Congress, 12. Dordrecht: Springer Science+Business Media B.V. 265p.

NAVIA, D. et al. 2011. First report of the red palm mite, Raoiella indica Hirst (Acari: Tenuipalpidae), in Brazil. Neotropical Entomology (Brasil) 40(3):409-411.

NUVOLONI, F. M. et al. 2014. Phytoseiidae mites associated with Hevea spp. from the Amazon region: a hidden diversity under the canopy of native trees. Systematics and Biodiversity 13(2):182-206.
OCHOA, R. et al. 2011. Brevipalpus phoenicis in Brazil. Who is it? In: Simpósio Brasileiro de Acarologia, 3. Campinas. Resumos... Campinas, SP, SIBAC.

OCHOA, R. et al. 2015. Brevipalpus mites in Brazil: a big challenge! In: Simpósio Brasileiro de Acarologia, 5. São José do Rio Preto. Resumos. São José do Rio Preto, SP. SIBAC.

PRASLIÈKA, J. et al. 2009. Predatory mites of the Phytoseiidae family in integrated and ecological pest management systems in orchards in Slovania. Biologia 64(5):959-961.

REIS, A. C. et al. 2010. Eriophyoid mites (Acari: Prostigmata: Eriophyoidea) from fruit trees in Northeastern Brazil - a new genus, three new species and a redescription. Zootaxa 2694:43-56.

SALAS, L. A.; OCHOA, R. 1986. El género Tenuipalpus Donn. en Costa Rica (Acari: Tenuipalpidae). Agronomía Costarricense 10(1-2):207-210.

SILVA, N. M. da; ADAIME, R.; ZUCCHI, R. A. (eds.). 2016. Pragas agrícolas e florestais na Amazônia. Brasília, Embrapa. $608 \mathrm{p}$.

SILVA, R. A.; JORDÃO, A. L. 2005. Pragas dos citros no Estado do Amapá. Revista Científica Eletrônica de Agronomia (Brasil) 7:277-280.

SINICO, T. E. et al. 2015. Marcadores moleculares para identificação e estudo de diversidade de Brevipalpus spp. (Acari: Tenuipalpidae): informações preliminares. In: Simpósio Brasileiro de Acarologia, 5. São José do Rio Preto. Resumos... São José do Rio Preto, SP, SIBAC.

SÓ GEOGRAFIA. VIRTUOUS TECNOLOGIA DA INFORMAÇÃO. 2019. Amapá. Disponível em: <http:// www.sogeografia.com.br/Conteudos/Estados/Amapa/> Acesso em: 26 mar. 2020.

SOUSA, M. S. M. et al. 2013. Mites (Arachnida: Acari) associated with hog plum trees (Spondias mombin L.) in the Brazilian Amazon. Biota Amazônia (Brasil) 3(1):177-180.

TANZINI, M. R. et al. 2000. An epizootic of Calacarus heveae (Acari: Eriophyidae) caused by Hirsutella thompsoni on rubber trees. Experimental and Applied Acarology 24(2):141-144.

TORO, S. S.; MESA, N. C. C. 2015. Parámetros poblacionales y comportamiento de Steneotarsonemus spinki Smiley (Acari: Tarsonemidae) en el cultivo de arroz. Protectio Acta Agronómica. 64:183-193.

VIEIRA, M. R. et al. 2009. Progênies de seringueira com potencial para conferir resistência a ácaros (Calacarus heveae Feres e Tenuipalpus heveae Baker). Ciência Rural (Brasil) 39(7):1953-1959,

YAMAMOTO, P. T.; BASSANEZI, R. B. 2003. Seletividade de produtos fitossanitários aos inimigos naturais de pragas dos citros. Laranja (Brasil) 24(2):353-382.

YANINEK, J. S.; MORAES, G. J. de. 1991. Mites in biological and integrated control of pests in agriculture. pp. 133-149. In: Dusbabek, F.; Bukva, V. (Eds.). Modern acarology. proceedings. International Congress of Acarology, 8. Prague: SPB Academic Publishing. 680p. 